\title{
The Dependence of the Internal Vibrational Frequencies of Liquid Water on Central Force Potentials
}

\author{
G. Jancsó* and P. Bopp \\ Max-Planck-Institut für Chemie (Otto Hahn Institut), Mainz \\ Z. Naturforsch. 38 a, 206-213 (1983); received November 23, 1982 \\ Dedicated to Professor Alfred Klemm on the occasion of his 70 th birthday
}

\begin{abstract}
The differences in the liquid phase internal vibrational frequencies of water, obtained from molecular dynamics (MD) simulations, between the two versions of the central force model of Rahman and Stillinger (CF1 and CF2) are investigated by employing the theory of Buckingham on solvent effects. It is found that the differences can be essentially accounted for by the different $\mathrm{O}-\mathrm{H}$ stretching cubic anharmonic force constants of $\mathrm{CF} 1$ and $\mathrm{CF} 2$. A significantly improved agreement between the results of MD simulations and spectroscopically observed liuqid phase frequencies could be achieved by using a harmonic force field, supplemented by a cubic stretching force constant, for the intramolecular interactions of water, and the CF2 potential for the intermolecular interactions.
\end{abstract}

\section{Introduction}

The central force potentials developed by Rahman, Stillinger and Lemberg [1] (called here CF1) and Stillinger and Rahman [2] (called here CF2) (see Appendix) have been very successful in describing structural and dynamic properties of liquid water under normal conditions [1,2], of aqueous electrolyte solutions [3-5] and even of certain types of ices [6]. Building on an initial set of central force interactions by Lemberg and Stillinger [7], which was, to our knowledge, never used for actual computer simulations, the two sets of potentials referred to above were constructed in such a way as to obtain structural and dynamical properties for liquid water as close to experimental results as possible. Indeed, the last version of these potentials is considered to be one of the best available models of liquid water [8].

In this communication, we will focus our attention on an aspect of these models which has, in our opinion, not yet been sufficiently considered. These models treat the water molecule as three distinct mass points, one oxygen and two hydrogens. The interatomic potentials were determined in such a way as to yield stable $\mathrm{H}_{2} \mathrm{O}$ molecules with an appropriate equilibrium geometry (which is in fact

\footnotetext{
* Permanent address: Central Research Institute for Physics of the Hungarian Academy of Sciences, Budapest, Hungary.

Reprint requests to Dr. P. Bopp, MPI für Chemie, Otto Hahn Institut, Saarstraße 23, D-6500 Mainz, FRG.
}

the gas phase geometry of water), and to permit oscillatory motions of the atoms about that equilibrium geometry. Within the framework of a central force model, the vibrational frequencies of the molecule are determined, in harmonic approximation, by only two independent force constants, the $\mathrm{O}-\mathrm{H}$ and the $\mathrm{H}-\mathrm{H}$ stretches. In both models discussed here, they were set to be $7.973 \mathrm{mdyn} / \AA$ and $1.7872 \mathrm{mdyn} / \AA$, respectively, leading to the vibrational frequencies, for a single molecule, reported in Table 1. Table 1 also lists the observed fundamentals and the usually adopted harmonic frequencies for the water molecule. The agreement between calculated and observed gas phase frequencies is seen to be not very satisfactory, especially the fact that the calculated symmetric stretching frequency $\omega_{1}$ is found to be higher than the calculated asymmetric frequency $\omega_{3}$, while the opposite is always observed in water. Furthermore, in spite of the fact that the harmonic stretching force constants are the same for both models (CF1 and CF2), very different oscillatory motions are observed in the liquid state.

These motions are usually studied by analyzing the Fourier transform of the normalized velocity autocorrelation function of the hydrogens as obtained from molecular dynamics simulations (cf. e.g. [3]). In their study of the CF1 model Rahman, Stillinger and Lemberg [1] found three broad bands in this Fourier transform, one centred at about $1475 \mathrm{~cm}^{-1}$, or shifted by about $+100 \mathrm{~cm}^{-1}$ with 
Table 1. Internal vibrational frequencies of $\mathrm{H}_{2} \mathrm{O}\left(\right.$ in $\left.\mathrm{cm}^{-1}\right)$.

\begin{tabular}{lllllll}
\hline Vibrational mode & $v_{\text {gas }}$ & $\omega_{\text {gas }}$ & $\omega_{\text {gas }}(\mathrm{CF})^{\mathrm{b}}$ & $v_{\text {liq }}{ }^{\mathrm{c}}$ & $v_{\text {liq }}(\mathrm{CF} 1)^{\mathrm{d}}$ & $v_{\text {liq }}(\mathrm{CF} 2)^{\mathrm{d}}$ \\
\hline sym. strech $\left(v_{1}, \omega_{1}\right)^{\mathrm{a}}$ & 3657 & 3832 & 4266 & $3345[14] ;$ & $\sim 3500$ & 4573 \\
& & & & $3225[13]$ & & 1593 \\
bend $\left(v_{2}, \omega_{2}\right)$ & $1595[9]$ & $1648[12]$ & $1369[1]$ & $1645[9]$ & $\sim 1475[1]$ & 1593 \\
asym. strech $\left(v_{3}, \omega_{3}\right)$ & 3756 & 3943 & 3805 & $3455[14] ;$ & $\sim 2900$ & 4164
\end{tabular}

a $v$ and $\omega$ denote fundamental and harmonic frequencies, respectively.

b Gas phase harmonic frequencies calculated from CF1 (CF2) model potential.

c The assignment of $v_{1}$ seems to be uncertain because of the Fermi resonance with $2 v_{2}$.

d $v_{\text {liq }}(\mathrm{CF} 1)$ and $v_{\text {lig }}(\mathrm{CF} 2)$ are the maxima of the Fourier transform of the velocity autocorrelation function of the hydrogens from MD simulations of water with CF1 and CF2 models, respectively.

respect to the frequency reported for the harmonic gas phase bending vibration in this model in Table 1, and two overlapping ones, centred at about $2900 \mathrm{~cm}^{-1}$ and $3500 \mathrm{~cm}^{-1}$, shifted by about $-840 \mathrm{~cm}^{-1}$ on the average with respect to the vapour phase stretching frequencies. In the case of CF2, the bands observed in the Fourier transform of the velocity autocorrelation function were centred at about $1600 \mathrm{~cm}^{-1}, 4160 \mathrm{~cm}^{-1}$ and $4570 \mathrm{~cm}^{-1}$ (this work) and the two latter ones were much sharper than the corresponding band in the CF1 case [3]. The calculated with respect to harmonic gas phase frequenvapour phase have to be compared with the experimentally observed ones (see Tables 1 and 3 ). In the work presented here, all frequency shifts were calculated with respect of harmonic gas phase frequencies, as the small additional shifts due to the classical anharmonic motions [10] in the gas phase can be neglected in this context.

It can be seen that, while in the case of CF1 the sign of the shifts is at least correct, this is not the case for the two high frequencies observed for the CF2 model.

In order to understand the origin of this quite different behaviour of these otherwise very similar models, we have undertaken a careful analysis of especially the intramolecular parts of the two sets of potentials, since some preliminary calculations indicated that the differences in the external interaction parts can not explain the different spectral features. In a subsequent step we then tested this concept by introducing a new potential for the intramolecular interactions. In order to obtain a more realistic behaviour of the water molecule vibrations, this potential was expressed as a power series in terms of interatomic stretches and bends, as it is usually done for potentials obtained from spectroscopic measurements. This part of the potential is thus no longer of the central force type, but also contains three body contributions.

At this stage of theoretical development, we feel that it is justified to study these effects within the framework of a classical theory, such as molecular dynamics, even though the vibrations have frequencies such that $h c \omega>k T$. As was pointed out earlier [7], the nature and the magnitude of the hydrogen bond distortions in the liquid can be analyzed and interpreted in terms of such classical models; their influence on the vibrational frequencies may thus be studied by these methods. Furthermore, as the anharmonicity constants (usually called $X_{\mathrm{e}}$ [10]) can safely be assumed not to vary significantly between the vapour and liquid phases [11] we are led to expect a generally analogueous behaviour of quantum frequency and classical frequency shifts between vapour and liquid, as calculated here.

Within this approach, it is thus worthwhile to try to improve the existing interaction potentials, aiming at a more realistic behaviour of the intramolecular vibrations. Such a model could subsequently be used to study e.g. the influence of solutes on the IR spectra of water in aqueous solutions more accurately than it has been possible in the past $[3,4]$.

\section{Perturbation Theory Treatment}

The vibrational frequencies of molecules in the liquid phase are different from those in the gaseous phase owing to the effect of intermolecular interactions on the properties of condensed phase molecules (see e.g. [15]). Buckingham [16] and others 
[16-18] have discussed the theory of the effect of intermolecular interactions on normal mode vibrational frequencies. In order to understand the reasons for the different spectral behaviour in the liquid phase of the two versions of the central force model potentials discussed above, - in spite of the fact that their harmonic stretching force constants are identical -, we decided to investigate, as a first approximation, the bond stretching vibrations of the water molecule as a diatomic oscillator within the framework of Buckingham's theory [16].

\section{A) Diatomic Oscillator Approximation}

A diatomic oscillator approximation seems especially justified if the molecule HDO is studied instead of $\mathrm{H}_{2} \mathrm{O}$, as in this case the $\mathrm{O}-\mathrm{H}$ and $\mathrm{O}-\mathrm{D}$ stretching vibrations can be treated in good approximation as two uncoupled diatomic oscillators. For the vapour-liquid frequency shifts of the fundamental absorption bands of a diatomic oscillator $(\Delta v)$, Buckingham [16] obtained the following expression by using perturbation theory and treating the anharmonicity and the intermolecular interaction energy in the liquid phase as perturbations to the harmonic oscillator

$$
\begin{aligned}
\Delta v=v_{\text {liq }}-v_{\text {vap }}= & \frac{B_{\mathrm{e}}}{h c \omega}\left\langle U^{\prime \prime}-3 \boldsymbol{a} U^{\prime}\right\rangle \\
& + \text { higher order terms }
\end{aligned}
$$

where $B_{\mathrm{e}}$ is the rotational constant $\left(h / 8 \pi^{2} \mu c r_{\mathrm{e}}^{2}\right), \mu$ being the reduced mass, $r_{\mathrm{e}}$ is the equilibrium distance, $\omega$ is the frequency of the harmonic oscillator (in $\mathrm{cm}^{-1}$ ). $U$ is the intermolecular interaction potential energy, expanded as a power series in the displacement of the nuclei from their equilibrium separation $\xi=\left(r-r_{\mathrm{e}}\right) / r_{\mathrm{e}}$

$$
U=U(\xi, \tau)=U_{\mathrm{e}}(\tau)+U^{\prime}(\tau) \xi+\frac{1}{2} U^{\prime \prime}(\tau) \xi^{2}+\ldots .
$$

$U^{\prime}$ and $U^{\prime \prime}$ are the derivatives of $U$ with respect to $\xi$ at the nuclear equilibrium separation $r_{\mathrm{e}}$, and $\tau$ represents the positions (and orientations) of all other molecules relative to a particular molecule. In (1) $\boldsymbol{a}$ is the cubic anharmonicity constant in the vapour phase and the brackets denote an averaging over all solvent configurations. The connection between the dimensionless constant $\boldsymbol{a}$ and the perhaps more familiar anharmonicity constant

$$
g \equiv 1 / 6\left(\frac{\partial^{3} V}{\partial r^{3}}\right)_{r=r_{\mathrm{e}}}
$$

is given by

$$
\boldsymbol{a}=\frac{4 B_{\mathrm{e}} r_{\mathrm{e}}^{3}}{h c \omega^{2}} g .
$$

For most liquids, under normal conditions, one has [16] $U^{\prime}<0, U^{\prime \prime}>0, a<0,(g<0)$ and, since $\left\langle U^{\prime \prime}\right\rangle$ usually is a much smaller than $3 \boldsymbol{a}\left\langle U^{\prime}\right\rangle$, a red shift $(\Delta v<0)$ for the stretching vibrations can be expected, in accord e.g. with experimental observations for $\mathrm{HDO}\left(\Delta v_{\mathrm{OH}}=-307 \mathrm{~cm}^{-1}, \Delta v_{\mathrm{OD}}=-227\right.$ $\left.\mathrm{cm}^{-1}[9]\right)$.

By differentiating the $V_{\mathrm{OH}}$ part of the central force potentials $\mathrm{CF} 1$ and $\mathrm{CF} 2$ [2], values of $-26.3 \mathrm{mdyn} / \AA^{2}$ and $+3.28 \mathrm{mdyn} / \AA^{2}$ are obtained for the cubic anharmonicity constant $(g)$, respectively ( $f_{\text {rrr }}$ in Table 2$)$. From (1) a red shift has to be expected for the CF1 potential and a blue shift for CF2. Also the magnitude of the shift should be larger in the former case than in the latter one. There are of course differences in the intermolecular interaction energies $(U)$ of the two model potentials, but they can be neglected for the arguments presented here. It should be mentioned that a positive $g$ value does not seem to be realistic from a physical point of view, since this would mean that the potential energy curve comes up inside the harmonic parabola as the bond stretches.

In order to obtain an estimate of the importance of the vibrational frequency shift due to the term $\left\langle U^{\prime \prime}\right\rangle$, a molecular dynamics simulation was run on HDO liquid. (We are aware of the fact that pure HDO liquid does not exist, but for the present

Table 2. Force constants (in mdyn/ $\AA$ ) and equilibrium geometry of the water molecule.

\begin{tabular}{llllllllll}
\hline Potential & $f_{r}$ & $f_{q}{ }^{\mathrm{a}}$ & $f_{r r}$ & $f_{r \alpha}$ & $f_{\alpha}$ & $f_{r r r}{ }^{\mathrm{b}}$ & $f_{q q q}{ }^{\mathrm{b}}$ & $r_{\mathrm{e}}(\AA)$ & $\alpha_{\mathrm{e}}$ \\
\hline Harmonic [12] & 8.454 & & -0.101 & 0.228 & 0.761 & & & 0.9572 & $104.52^{\circ}$ \\
CF1 [1] & 7.973 & 1.7872 & & & & -26.3 & -0.863 & 0.9584 & $104.45^{\circ}$ \\
CF2 [2] & 7.973 & 1.7872 & & & & +3.28 & -0.817 & \\
\hline
\end{tabular}

\footnotetext{
a $\mathrm{H}-\mathrm{H}$ stretching force constant.

b These anharmonic force constants are given in $\mathrm{mdyn} / \AA^{2}$.
} 
Table 3. Vapour-liquid frequency shifts $\left(\right.$ in $\mathrm{cm}^{-1}$ ) of internal vibrations of water obtained from MD simulations.

\begin{tabular}{|c|c|c|c|c|c|c|c|}
\hline \multirow[t]{2}{*}{ Molecule } & \multicolumn{3}{|c|}{ Molecular Dynamics Simulation } & \multicolumn{3}{|c|}{ Vapour-liquid frequency shifts ${ }^{e}$} & \multirow[t]{2}{*}{ Ref. } \\
\hline & $\begin{array}{l}\text { intermolecular } \\
\text { interactions }\end{array}$ & $\begin{array}{l}\text { intramolecular } \\
\text { interactions }\end{array}$ & $\begin{array}{l}\text { average } \\
\text { temperature }(\mathrm{K})\end{array}$ & $\Delta v_{1}$ & $\Delta v_{2}$ & $\Delta v_{3}$ & \\
\hline $\begin{array}{l}\mathrm{HDO} \\
\mathrm{HDO} \\
\mathrm{H}_{2} \mathrm{O} \\
\mathrm{H}_{2} \mathrm{O} \\
\mathrm{H}_{2} \mathrm{O}\end{array}$ & $\begin{array}{l}\text { CF2 } \\
\text { CF2 } \\
\text { CF1 } \\
\text { CF2 } \\
\text { CF2 }\end{array}$ & $\begin{array}{l}\text { Harmonic }[12] \\
\text { Harmonic }+f_{r r r} \text { b } \\
\text { CF1 } \\
\text { CF2 } \\
\text { Harmonic }[12]\end{array}$ & $\begin{array}{l}319 \\
289 \\
295 \\
302 \\
300\end{array}$ & $\begin{array}{r}59^{\mathrm{a}} \\
-201 \\
\sim-770 \\
306 \\
\ldots \mathrm{d}\end{array}$ & $\begin{array}{r}69 \\
64 \\
106 \\
225 \\
76\end{array}$ & $\begin{array}{r}49 \\
-141 \\
\sim-900 \\
358 \\
\ldots \mathrm{d}\end{array}$ & $\begin{array}{l}c \\
c \\
{[1]} \\
c \\
c\end{array}$ \\
\hline
\end{tabular}

a Gas frequencies for HDO: $\omega_{1}=3889 \mathrm{~cm}^{-1}, \omega_{2}=1446 \mathrm{~cm}^{-1}, \omega_{3}=2824 \mathrm{~cm}^{-1}$. Observed vapour-liqud frequency shifts: $\Delta v_{1}=-307 \mathrm{~cm}^{-1}, \Delta v_{2}=45 \mathrm{~cm}^{-1}, \Delta v_{3}=-227 \mathrm{~cm}^{-1}[9]$.

b $f_{\text {rrr }}=-9.977 \mathrm{mdyn} / \AA^{2}[12]$.

c This work, the error of the calculated frequencies is $\pm 15 \mathrm{~cm}^{-1}$.

d There is one wide band in the Fourier transform with a maximum at $3923 \mathrm{~cm}^{-1}$.

e The shifts are calculated with respect to the harmonic gas phase frequencies.

purpose it does not cause any problems.) For the internal (intramolecular) interactions, a harmonic force field in $\mathrm{O}-\mathrm{H}$ and $\mathrm{O}-\mathrm{D}$ bond stretches and $\mathrm{H}-\mathrm{O}-\mathrm{D}$ bond angle displacements was used [12] (see Table 2), the external (intermolecular) interactions were taken from the CF2 model potential, which, as has been pointed out above, is considered to be a very reliable one. A suitably modified version of the program used in previous simulations with the central force model for water $[3,4]$ was employed here; the reader is referred to these papers for further details. After equilibration, a run of 1000 time steps was generated (average temperature $319 \mathrm{~K}$ ), and the velocity autocorrelation functions for $\mathrm{H}$ and $\mathrm{D}$ and their Fourier transforms were evaluated. It might be noted that it is also possible to evaluate the velocity autocorrelation function of all hydrogens $(\mathrm{H}$ and $\mathrm{D})$; the positions of the maxima in the Fourier transform of this function are the same as the ones obtained from the Fourier transforms of the individual (H or D) velocity autocorrelation functions.

The vapour-liquid frequency shifts obtained are $+59 \mathrm{~cm}^{-1}$ for the $\mathrm{O}-\mathrm{H},+49 \mathrm{~cm}^{-1}$ for the $\mathrm{O}-\mathrm{D}$ stretches (and incidentally, $+69 \mathrm{~cm}^{-1}$ for the $\mathrm{H}-\mathrm{O}-\mathrm{D}$ bend), which gives an average value of $0.247 \mathrm{mdyn} \cdot \AA$ for $\left\langle U^{\prime \prime}\right\rangle$. The corresponding change in the harmonic stretching force constant $\left(\left\langle U^{\prime \prime}\right\rangle / r_{\mathrm{e}}^{2}\right)$ amounts to $0.26 \mathrm{mdyn} / \AA$; this small value also indicates that the main part of the vapour-liquid frequency shift arises from the second term in (1), containing the anharmonicity constant $\boldsymbol{a}$. As the same order of magnitude for $\left\langle U^{\prime \prime}\right\rangle / r_{\mathrm{e}}^{2}$ is to be expected for the CF1 potential, we conclude that the difference in the anharmonicity of the $\mathrm{O}-\mathrm{H}$ stretching potential between the $\mathrm{CF} 1$ and $\mathrm{CF} 2$ versions is responsible for the different vapour-liquid shifts of the stretching frequencies obtained from the MD simulations.

On the other hand, it is clear that a proper explanation of the differences observed for the bending frequencies (cf. Tables 1 and 3) can not be achieved by the extremly simplified treatment presented here. Therefore, a more complete treatment of the case of triatomic molecules will be worked out in the next section.

\section{B) Triatomic Molecule Treatment}

For $\mathrm{H}_{2} \mathrm{O}$ and $\mathrm{D}_{2} \mathrm{O}$, the vapour-liquid vibrational frequency shifts can be written, according to Buckingham [16], to first approximation as

$$
\begin{aligned}
& \Delta v_{1}=\frac{1}{8 \pi^{2} c^{2} \omega_{1}^{2}}\{\left\langle U_{11}^{\prime \prime}\right\rangle-\frac{3}{2 \pi^{2} c^{2}} \\
&\left.\cdot\left(\left\langle U_{1}^{\prime}\right\rangle \frac{V_{111}}{\omega_{1}^{2}}+\left\langle U_{2}^{\prime}\right\rangle \frac{V_{211}}{\omega_{2}^{2}}\right)\right\}
\end{aligned}
$$

(symmetric stretching),

$$
\begin{array}{r}
\Delta v_{2}=\frac{1}{8 \pi^{2} c^{2} \omega_{2}^{2}}\left\{\left\langle U_{22}^{\prime \prime}\right\rangle-\frac{3}{2 \pi^{2} c^{2}}\right. \\
\left.\cdot\left(\left\langle U_{1}^{\prime}\right\rangle \frac{V_{122}}{\omega_{1}^{2}}+\left\langle U_{2}^{\prime}\right\rangle \frac{V_{222}}{\omega_{2}^{2}}\right)\right\}
\end{array}
$$

(bending),

$$
\begin{aligned}
& \Delta v_{3}=\frac{1}{8 \pi^{2} c^{2} \omega_{3}^{2}}\left\{\left\langle U_{33}^{\prime \prime}\right\rangle-\frac{3}{2 \pi^{2} c^{2}}\right. \\
&\left.\cdot\left(\left\langle U_{1}^{\prime}\right\rangle \frac{\mathrm{V}_{133}}{\omega_{1}^{2}}+\left\langle U_{2}^{\prime}\right\rangle \frac{V_{233}}{\omega_{2}^{2}}\right)\right\}
\end{aligned}
$$

(asymmetric stretching) 
(for HDO, the corresponding formulae are more complicated because of the lack of molecular symmetry). In these expressions, $U_{i}^{\prime}=\left(\partial U / \partial Q_{i}\right)_{\mathrm{e}}$ and $U_{i j}^{\prime \prime}$ are derivatives of the interaction potential with respect to normal coordinates; the brackets denote, as before, an averaging over all solvent configurations. $U$ is written as a series expansion

$$
\begin{aligned}
U & =U\left(Q_{1}, Q_{2}, Q_{3}, \tau\right) \\
& =U_{\mathrm{e}}+\sum_{i=1}^{3} U_{i}^{\prime} Q_{i}+\frac{1}{2} \sum_{i, j} U_{i j}^{\prime \prime} Q_{i} Q_{j}+\ldots
\end{aligned}
$$

There are no $\left\langle U_{3}^{\prime}\right\rangle$ terms in $(4 a)-(4 c)$ because of symmetry requirements. The $V_{i j k}$ terms are the anharmonic force constants, obtained when the intramolecular potential energy $(V)$ is expanded in terms of normal coordinates

$$
\begin{aligned}
V= & 2 \pi^{2} c^{2}\left(\omega_{1}^{2} Q_{1}^{2}+\omega_{2}^{2} Q_{2}^{2}+\omega_{3}^{2} Q_{3}^{2}\right)+V_{111} Q_{1}^{3} \\
& +V_{122} Q_{1} Q_{2}^{2}+V_{133} Q_{1} Q_{3}^{2}+V_{211} Q_{1}^{2} Q_{2} \\
& +V_{222} Q_{2}^{3}+V_{233} Q_{2} Q_{3}^{2} \\
& + \text { higher order terms. }
\end{aligned}
$$

It is more convenient to write the potential energy in wavenumber units as an expansion in the dimensionless normal coordinates $\left(x_{i}\right)[9]$

$$
\begin{aligned}
V / h c= & \frac{1}{2}\left(\omega_{1} x_{1}^{2}+\omega_{2} x_{2}^{2}+\omega_{3} x_{3}^{2}\right)+k_{111} x_{1}^{3} \\
& +k_{122} x_{1} x_{2}^{2}+k_{133} x_{1} x_{3}^{2}+k_{211} x_{1}^{2} x_{2} \\
& +k_{222} x_{2}^{3}+k_{233} x_{2} x_{3}^{2} \\
& + \text { higher order terms, }
\end{aligned}
$$

where $k_{i j k}$ is given in $\mathrm{cm}^{-1}$ and $x_{i}=2 \pi\left(c \omega_{i} / h\right)^{1 / 2} Q_{i}$. It should also be mentioned that in (6) and (7) terms containing odd powers of $Q_{3}$ or $x_{3}$ are missing because of the $C_{2 \mathrm{v}}$ symmetry of water molecule.

In order to estimate the contributions of the different terms in $(4 a)-(4 c)$ to the vapour-liquid shifts of vibrational frequencies of water, it is necessary first to evaluate the cubic anharmonic constants $\left(V_{i j k}, k_{i j k}\right)$ in terms of internal coordinates. The vibrational potential functions of type $\mathrm{CF} 1$ and $\mathrm{CF} 2$ can be expanded in terms of internal coordinates in the following way:

$$
\begin{aligned}
V= & \frac{1}{2} f_{r}\left(\Delta r_{1}^{2}+\Delta r_{2}^{2}\right)+\frac{1}{2} f_{q} \Delta q^{2} \\
& +\frac{f_{r r r}}{r_{e}}\left(\Delta r_{1}^{3}+\Delta r_{2}^{3}\right)+\frac{f_{q q q}}{q_{e}} \Delta q^{3}+\ldots,
\end{aligned}
$$

where $\Delta r_{1}\left(\Delta r_{2}\right)$ and $\Delta q$ represent the displacements of $\mathrm{O}-\mathrm{H}$ and $\mathrm{H}-\mathrm{H}$ bond lengths, respectively. The values of the anharmonic force constants for the two versions of the central force potential obtained by differentiating $V_{\mathrm{OH}}$ and $V_{\mathrm{HH}}$ are listed in Table 2. It can be seen that the $f_{q q q}$ force constants are nearly identical for $\mathrm{CF} 1$ and $\mathrm{CF} 2$; therefore it is difficult to understand at first sight why the vapour-liquid shifts of the bending frequencies are so different $\left(+106 \mathrm{~cm}^{-1}\right.$ in CF 1 vs. $+225 \mathrm{~cm}^{-1}$ in CF2).

The anharmonic potential terms $V_{i j k}$ can now be evaluated as functions of the quadratic and higher order force constants by the transformation $[12,20]$ shown in matrix form in Table 4 using the notation of Eq. (23) in [20]. Numerical values for these coefficients can be derived from the eigenvector matrix elements of the harmonic vibrational problem adequate for the central force fields, i.e. using two $\mathrm{O}-\mathrm{H}$ stretches and the $\mathrm{H}-\mathrm{H}$ stretch as coordinates and the force constants reported in Table 2, by methods described in $[12,20]$. The numerical values are listed along with the eigenvector elements in Table 5. It is interesting to note that there is much

Table 4. Matrix representation of the cubic potential constants for central potential of water ${ }^{\mathrm{a}}$.

$\left[\begin{array}{l}V_{111} \\ V_{122} \\ V_{133} \\ V_{211} \\ V_{222} \\ V_{233}\end{array}\right]=\left[\begin{array}{cccc}\alpha^{2} \lambda^{\mathrm{b}} & 0 & \lambda^{3} & \delta^{3} \\ \beta(\beta \lambda+2 \alpha \mu) & 0 & 3 \lambda \mu^{2} & 3 \delta \varepsilon^{2} \\ \gamma(\gamma \lambda+2 \alpha \nu) & \delta \sigma^{2} & 3 \lambda v^{2} & 0 \\ \alpha(\alpha \mu+2 \beta \lambda) & 0 & 3 \lambda^{2} \mu & 3 \delta^{2} \varepsilon \\ \beta^{2} \mu & 0 & \mu^{3} & \varepsilon^{3} \\ \gamma(\gamma \mu+2 \beta v) & \varepsilon \sigma^{2} & 3 \mu v^{2} & 0\end{array}\right]\left[\begin{array}{c}\frac{f_{r}}{r_{\mathrm{e}}} \\ \frac{f_{q}}{q_{\mathrm{e}}} \\ \frac{2 f_{r r r}}{r_{\mathrm{e}}} \\ \frac{f_{q q q}}{q_{\mathrm{e}}}\end{array}\right]$

a Units: $\left[V_{i j k}\right]=\mathrm{g}^{-1 / 2} \mathrm{~cm}^{-1} \mathrm{~s}^{-2} \mathrm{~N}^{-3 / 2} ;[\alpha, \beta, \ldots]=\mathrm{amu} ;[f]$ $=\mathrm{dyn} / \mathrm{cm} ;\left[r_{\mathrm{e}}, q_{\mathrm{e}}\right]=\mathrm{cm}$.

$\mathrm{b}$ The symbols $\alpha, \beta$, etc. are defined in Eq. (23) of Ref. [20], the numerical values are given in Table 5 .

Table 5. The eigenvector matrix elements (in $a m u^{-1 / 2}$ ) and the transformation coefficients (in $\mathrm{amu}$ ) for central force water.

\begin{tabular}{llll}
\hline & $Q_{1}$ & $Q_{2}$ & $Q_{3}$ \\
\hline$\Delta r_{1}{ }^{\text {a }}$ & 0.708 & 0.133 & -0.731 \\
$\Delta r_{2}$ & 0.708 & 0.133 & 0.731 \\
$\Delta q$ & 1.234 & -0.679 & 0 \\
$\alpha=-0.0933$ & $\beta=0.7376$ & $\gamma=-0.0414$ & $\lambda=0.7145$ \\
$\mu=0.1327$ & $v=0.7327$ & $\delta=1.2439$ & $\varepsilon=-0.6939$ \\
& & & $\sigma=-0.8322$
\end{tabular}

a $\Delta r_{1}\left(\Delta r_{2}\right)$ and $\Delta q$ are the $\mathrm{O}-\mathrm{H}$ and $\mathrm{H}-\mathrm{H}$ stretching coordinates, respectively. 
more mixing between the symmetric stretching and "bending" modes in the case of central force potentials than in the case of the usual spectroscopic potentials (two stretches, one bend).

\section{Discussion}

The contributions from the different force constants (in internal coordinates, see Table 2) to the anharmonic potential constants $k_{i j k}$ of (7) are listed in Table 6. The cubic constants of the central force potentials in terms of the normal coordinates, which play a central role in the determination of the vapour-liquid shifts of the internal vibrations (4), are given in Table 7 .

It is an interesting point that in some cases (e.g. $\left.V_{112}\right)$ there is a significant contribution from the quadratic force constants to the anharmonic potential constants, which originates from the nonlinear dependence of the internal coordinates on the normal coordinates [21]. The differences in the signs of the $V_{111}, V_{211}, V_{133}$ and $V_{233}$ cubic constants for CF 1 and $\mathrm{CF} 2$ are in agreement with the red and blue shifts of the symmetric and asymmetric stretching frequencies observed in the MD calculations (cf. Table 3). The magnitude of $V_{133}$ is much larger than that of $V_{111}$, which explains the larger shift of the asymmetric stretching frequency compared to that of the symmetric stretching frequency. It can be seen that the magnitude of the contribution of $f_{r r r}$ to $V_{122}$ is significantly smaller for CF2 than for CF1. This might account for a large part of the difference between the bending vibrational frequencies of the two models in the liquid state. On the other hand, the different signs of $V_{122}$ for CF 1 and CF2 seem to indicate that higher order terms in (4b) may also contribute to the vapour-liquid shift of the bending vibrational frequency.

From Table 7, the second term in $(4 \mathrm{~b})$ containing $V_{222}$ seems to be much less important than the first term; this shows that a term in $\left\langle U_{1}^{\prime}\right\rangle$ can contribute significantly to $\Delta v_{2}$ through the anharmonicity constant $V_{122}$. The importance of this point has already

Table 6. Contributions from internal force constants to cubic constants $k_{i j k}$ (in $\mathrm{cm}^{-1}$ ).

\begin{tabular}{|c|c|c|c|c|c|c|c|c|c|c|c|c|}
\hline & \multicolumn{2}{|l|}{$k_{111}$} & \multicolumn{2}{|l|}{$k_{122}$} & \multicolumn{2}{|l|}{$k_{133}$} & \multicolumn{2}{|l|}{$k_{211}$} & \multicolumn{2}{|l|}{$k_{222}$} & \multicolumn{2}{|l|}{$k_{233}$} \\
\hline & $\mathrm{CF} 1$ & CF2 & CF 1 & $\mathrm{CF} 2$ & CF1 & CF2 & CF1 & CF2 & CF 1 & CF2 & CF 1 & CF2 \\
\hline$f_{r}$ & \multicolumn{2}{|c|}{2} & \multicolumn{2}{|c|}{340} & \multicolumn{2}{|c|}{1} & \multicolumn{2}{|c|}{-51} & \multicolumn{2}{|c|}{117} & \multicolumn{2}{|c|}{-26} \\
\hline$f_{q}$ & \multicolumn{2}{|c|}{0} & \multicolumn{2}{|c|}{0} & \multicolumn{2}{|c|}{20} & \multicolumn{2}{|c|}{0} & \multicolumn{2}{|c|}{0} & \multicolumn{2}{|c|}{-20} \\
\hline$f_{r r r}$ & -678 & 85 & -219 & 27 & -2400 & 299 & -667 & 83 & -24 & 3 & -787 & 98 \\
\hline$f_{q q q}$ & -59 & -56 & -171 & -162 & 0 & 0 & 174 & 164 & 56 & 53 & 0 & 0 \\
\hline Total & -735 & 31 & -50 & 205 & -2379 & 320 & -544 & 196 & 149 & 173 & -833 & 52 \\
\hline
\end{tabular}

Table 7. Cubic constants in terms of normal coordinates ${ }^{\text {a }}$ for central force potentials of water $\left(V_{i j k} \times 10^{-15}\right)$.

\begin{tabular}{|c|c|c|c|c|c|c|c|c|c|c|c|c|}
\hline & \multicolumn{2}{|l|}{$V_{111}^{\mathrm{b}, \mathrm{c}}$} & \multicolumn{2}{|l|}{$V_{122}$} & \multicolumn{2}{|l|}{$V_{133}$} & \multicolumn{2}{|l|}{$V_{211}$} & \multicolumn{2}{|l|}{$V_{222}$} & \multicolumn{2}{|l|}{$V_{233}$} \\
\hline & $\mathrm{CF} 1$ & $\mathrm{CF} 2$ & $\mathrm{CF} 1$ & $\mathrm{CF} 2$ & $\mathrm{CF} 1$ & CF2 & $\mathrm{CF} 1$ & CF2 & $\mathrm{CF} 1$ & $\mathrm{CF} 2$ & $\mathrm{CF} 1$ & CF2 \\
\hline$\frac{f_{r}}{r_{\mathrm{e}}}$ & \multicolumn{2}{|c|}{5} & \multicolumn{2}{|c|}{308} & \multicolumn{2}{|c|}{2} & \multicolumn{2}{|c|}{-81} & \multicolumn{2}{|c|}{60} & \multicolumn{2}{|c|}{-37} \\
\hline$\frac{f_{q}}{q_{\mathrm{e}}}$ & \multicolumn{2}{|c|}{0} & \multicolumn{2}{|c|}{0} & \multicolumn{2}{|c|}{51} & \multicolumn{2}{|c|}{0} & \multicolumn{2}{|c|}{0} & \multicolumn{2}{|l|}{-28} \\
\hline$\frac{2 f_{r r r}}{r_{\mathrm{e}}}$ & -1919 & 239 & -196 & 25 & -6053 & 755 & -1069 & 133 & -13 & 2 & -1124 & 140 \\
\hline$\frac{f_{q 4 q}}{q_{\mathrm{e}}}$ & -166 & -157 & -155 & -147 & 0 & 0 & 278 & 263 & 29 & 27 & 0 & 0 \\
\hline Total & -2080 & 87 & -43 & 186 & -6000 & 808 & -872 & 315 & 76 & 89 & -1189 & 75 \\
\hline
\end{tabular}

a See Eq. (6).

c The relationship between $\dot{V}_{i j k}$ and $k_{i j k}$ is given by $V_{i j k}=k_{i j k}\left(\omega_{i} \omega_{j} \omega_{k}\right)^{1 / 2} / 9.8567 \cdot 10^{-7}$. 
been emphasized by Buckingham [16]. Also, the contribution of the $f_{q q q}$ force constant to the different cubic potential constants (see Tables 6 and 7) is sizeable in some cases (e.g. $V_{122}, V_{211}$ ), however, its effect on the differences between the vibrational frequency shifts of CF1 and CF2 is negligible. It is also clear that the differences between the shifts for $\mathrm{CF} 1$ and $\mathrm{CF} 2$ central force potential can not entirely be ascribed to the differences in the $V_{i j k}$ terms, since the external interactions are not exactly identical for the two versions.

By using the value of $0.247 \mathrm{mdyn} \cdot \AA$ for $\left\langle U^{\prime \prime}\right\rangle$ obtained in the diatomic oscillator approximation and the transformation between normal coordinates and internal coordinates, the harmonic shift corresponding to the $\left\langle U_{11}^{\prime \prime}\right\rangle$ and $\left\langle U_{33}^{\prime \prime}\right\rangle$ terms can be estimated for water with harmonic intramolecular potential. We obtained a value of $+64 \mathrm{~cm}^{-1}$ for $\Delta v_{1 \text { harm }} \cong \Delta v_{3 \text { harm }}$, which seems to be quite reasonable and suggests that if the gas phase vibrational motions were purely harmonic, the vapour-liquid shifts would be small and to the blue.

To have a further check on this point, a MD simulation was performed on $\mathrm{H}_{2} \mathrm{O}$ with harmonic intramolecular and $\mathrm{CF} 2$ intermolecular potential. Using the techniques described above, a wide band with an unresolved peak centred at $3923 \mathrm{~cm}^{-1}$ (see Table 3) was obtained. It should be mentioned that in this case the shifts can not be expected to correspond exactly to the predicted harmonic shifts because of the contributions from the quadratic force constants to the cubic $V_{i j k}$ constants (see Table 7). In spite of this, the shifts calculated from MD seem to have the right order of magnitude.

It can be concluded from the above discussion that $f_{r r r}$ can be expected to have the largest influence on the vibrational frequency shifts on condensation in the case of water. Therefore we carried out a preliminary MD simulation on HDO liquid, using the harmonic force field of Kuchitsu and Morino [12] and their value of $-9.977 \mathrm{mdyn} / \AA^{2}$ for the cubic stretching force constant $f_{r r r}$. It can be seen from Table 3 that the introduction of this cubic force constant improved significantly the agreement between calculated and observed liquid phase frequencies for the stretching vibrations, while the bending frequency shift did not change significantly. This can be accounted for by the very small coupling between the stretching and bending motions in this molecule.

\section{Conclusion}

It has been demonstrated that the differences in the liquid phase intramolecular vibrational frequencies of the two versions of the central force model of water [1, 2], as obtained from MD simulations, are determined mainly by their different anharmonicities in the intramolecular parts of the potential, and are thus probably largely governed by the distortions of the $\mathrm{O}-\mathrm{H}$ bond in the liquid. The construction of a model potential which is suitable for a more realistic study of the internal vibrational frequencies (e.g. the effects of solutes on these) and at the same time maintains the good agreement with the experimentally observed structural and dynamical properties of liquid water thus requires the inclusion of some carefully selected anharmonic force constants into the internal part of the potential. Further studies along these lines are in progress; results of these investigations will be published separately.

\section{Acknowledgement}

The authors are indebted to Drs. K. Heinzinger, W. Dietz, Gy. I. Szász, and R. Sonnenschein for stimulating discussions. One of us (G. J.) thanks the Max-Planck-Gesellschaft for a fellowship, and financial support by the Deutsche Forschungsgemeinschaft is also acknowledged.

\section{Appendix}

For the convenience of the reader, the two versions of the central force model potential of Rahman and Stillinger $[1,2]$ are given below, (energy in $\mathrm{kcal} / \mathrm{mol}$ and length in $\AA$ ):

$$
\begin{aligned}
\mathrm{CF} 1: & \\
V_{\mathrm{OO}}(r)= & 144.538 / r+23401.9 / r^{8.3927} \\
V_{\mathrm{OH}}(r)= & -72.269 / r+2.6677 / r^{14.97} \\
& -6 /(1+\exp (5.49305(r-2.2))), \\
V_{\mathrm{HH}}(r)= & 36.1345 / r+20 /(1+\exp (40(r-2))) \\
& -17.03002 \exp \left(-7.60626(r-1.4525)^{2}\right), \\
\mathrm{CF} 2: \quad & 144.538 / r+26758.2 / r^{8.8591} \\
V_{\mathrm{OO}}(r) & -0.25 \exp \left(-4(r-3.4)^{2}\right) \\
& -0.25 \exp \left(-1.5(r-4.5)^{2}\right), \\
V_{\mathrm{OH}}(r)= & -72.269 / r+6.23403 / r^{9.19912} \\
& -10 /(1+\exp (40(r-1.05))) \\
& -4 /(1+\exp (5.49305(r-2.2))), \\
& -17 \exp \left(-7.62177(r-1.45251)^{2}\right) .
\end{aligned}
$$


[1] A. Rahman, F. H. Stillinger, and F. Lemberg, J. Chem. Phys. 63, 5223 (1975).

[2] F. H. Stillinger and A. Rahman, J. Chem. Phys. 68, 666 (1978).

[3] P. Bopp, W. Dietz, and K. Heinzinger, Z. Naturforsch. 34 a, 1424 (1979).

[4] W. Dietz, W. O. Riede, and K. Heinzinger, Z. Naturforsch. 37 a, 1038 (1982).

[5] G. Pálinkás, T. Radnai, W. Dietz, Gy. I. Szász, and K. Heinzinger, Z. Naturforsch. 37 a, 1049 (1982); Gy. I. Szász, W. Dietz, K. Heinzinger, G. Pálinkás, and T. Radnai, Chem. Phys. Lett. 92, 388 (1982).

[6] M. D. Morse and S. A. Rice, J. Chem. Phys. 76, 650 (1982).

[7] H. L. Lemberg and F. H. Stillinger, J. Chem. Phys. 62, 1677 (1975).

[8] W. L. Jorgensen, J. Chem. Phys. 77, 4156 (1982).

[9] D. Eisenberg and W. Kauzmann, The Structure and Properties of Water, Oxford University Press, London 1969.

[10] G. Herzberg, Molecular Spectra and Molecular Structure, Vol. I Ch. III and Vol. II Ch. II, D. van Nostrand Co., Toronto 1945 and 1950.

[11] G. Jancsó and W. A. Van Hook, J. Mol. Struct. 48, 107 (1978) and references therein.
[12] K. Kuchitsu and Y. Morino, Bull. Chem. Soc. Japan 38, 814 (1965).

[13] J. R. Scherrer, M. K. Go, and S. Kint, J. Phys. Chem. 78, 1304 (1974).

[14] W. F. Murphy and H. J. Bernstein, J. Phys. Chem. 76, 1147 (1972).

[15] N. G. Bakhshiev, Spectroscopy of Intermolecular Interactions, (in Russian), Nauka, Leningrad 1972.

[16] A. D. Buckingham, Proc. Roy. Soc. London A 248, (1958); ibid, A 255, 32 (1960); Trans. Faraday Soc. 56, $753(1960)$

[17] A. Foeldes and C. Sandorfy, J. Mol. Spectrosc. 20, 262 (1966); G. Durocher and C. Sandorfy, J. Mol. Spectrosc. 22, 347 (1967).

[18] N. G. Bakhshiev and O. P. Girin, Opt. Spectrosc. 41, 125 (1976).

[19] D. M. Denisson, Rev. Mod. Phys. 12, 175 (1940).

[20] K. Kuchitsu and L. S. Bartell, J. Chem. Phys. 36, 2640 (1962).

[21] K. Kuchitsu and Y. Morino, Bull. Chem. Soc. Japan 38, 805 (1965). 